\title{
Die Ära der Entwicklung: Das Ende eines Mythos
}

\section{Von Wolfgang Sachs}

$\mathrm{M}$ it der Coronakrise und ihren heute bereits absehbaren verheerenden Auswirkungen auf den globalen Süden taucht plötzlich ein Wort wieder auf, das fast in der Versenkung verschwunden zu sein schien: Entwicklung. Denn genau das ist die Frage - ob es dergleichen, und zwar inzwischen nicht mehr nur für den Süden, sondern auch für den gesamten Globus, noch einmal geben kann, oder ob wir eine weiter zunehmende, fundamentale Disruption der Welt erleben.

Allerdings, daran gilt es zu erinnern, war Entwicklung von Beginn an ein Plastikwort gewesen, ein leerer Begriff mit ungewisser positiver Bedeutung. Gleichwohl hat sich Entwicklung als Weltperspektive gehalten, denn sie ist heute in ein internationales Geflecht von Institutionen eingebettet, von den Vereinten Nationen über Ministerien bis hin zu NGOs. Heute gehört Entwicklung zu jenen Zombie-Kategorien, die schon längst verwest sind, aber dennoch als abgedroschene Utopie allenthalben herumgeistern. Scheinbar schon lange zu Grabe getragen, treibt der Begriff wie ein Gespenst seinen Unfug auch noch in der Gegenwart. Und jetzt, in der zweiten Jahreshälfte 2020, kommt das Schlüsselwort wieder zu zweifelhafter Ehre, jährt sich doch zum fünften Mal die Verkündigung der päpstlichen Enzyklika „Laudato si'“, die Agenda 2030 der Vereinten Nationen mit den Sustainable Development Goals und das Klimaschutzabkommen von Paris. Damit stellt sich die entscheidende Frage: Sind diese internationalen Stellungnahmen überhaupt noch der Entwicklung verpflichtet? Oder kann man sie im Gegenteil als Ausweis des Post-Development-Denkens betrachten?

\section{Anno 1988 - eine Rückblende}

Eine Rückblende: Was waren wir damals, vor jetzt gut 30 Jahren, naiv und auch ein wenig großspurig, das "Ende des Entwicklungszeitalters" auszurufen! Im Herbst des Jahres 1988 an der Pennsylvania State University im Haus von Barbara Duden, wo wir unsere Freunde zu Living Room Consultations versammelt haben, fassten wir den vollmundigen Plan, ein „Development Dictionary" herauszubringen. Auf die Spur gesetzt von Ivan Illich, der einst den Plan hatte, eine "Archäologie der modernen Gewissheiten" zu verfassen, 
wollten wir uns an den Schlüsselbegriff der Entwicklung heranmachen, der, wie wir damals sagten, als Ruine in der intellektuellen Landschaft herumgestanden hat. ${ }^{1}$

Man erinnere sich: In der zweiten Hälfte des 20. Jahrhunderts thronte ganz selbstverständlich das Leitbild der Entwicklung wie ein mächtiger Herrscher über den Nationen der südlichen Hemisphäre. Es war das weltpolitische Programm der postkolonialen Epoche. Unschuldig kam der Begriff daher, aber er hatte es in sich: Als eine Art von mentaler Infrastruktur hat er den Weg für die imperiale Macht des Westens über die Welt geebnet. Wie im Westen, so auch auf Erden, so lautete, knapp gefasst, die Botschaft der Entwicklung. ${ }^{2}$

Wann aber hat das Entwicklungszeitalter angefangen? In unserem Development Dictionary stilisierten wir Präsident Harry S. Truman als Bösewicht. Und tatsächlich hatte dieser am 20. Januar 1949 in seiner Antrittsrede vor dem amerikanischen Kongress die Heimat von mehr als der Hälfte der Weltbevölkerung als "unterentwickelte Gebiete" bezeichnet. Mit dieser Rede war das Entwicklungszeitalter eröffnet - jene Periode der Weltgeschichte, die auf das unselige Kolonialzeitalter der europäischen Mächte folgte. Das Entwicklungszeitalter dauerte etwa 40 Jahre und wurde von der Epoche der Globalisierung abgelöst. Und derzeit erleben wir offensichtlich wieder eine Zäsur: den Aufschwung des Nationalpopulismus, als Backlash gegen die Globalisierung, der durch die Coronakrise enorm verstärkt werden dürfte. Worin aber bestand die Idee der Entwicklung? Es empfiehlt sich, dabei vier zentrale Aspekte zu unterscheiden.

Chronopolitisch, so die Idee, scheinen sich alle Völker auf dem Erdkreis auf einer einzigen Bahn vorwärts zu bewegen, im Sinne einer allgemein gültigen Aufstiegsentwicklung. Die imaginierte Zeit ist linear; indem sich diese ausbreitet, werden Völker wie etwa die Rajasthanis in Indien oder die Aymara in Peru unausweichlich in die Perspektive des weltweiten Fortschritts gezogen.

Geopolitisch geben die führenden Läufer, die entwickelten Nationen, den Weg der Entwicklung für die Nachzügler-Länder vor. Die vormals verwirrende Vielfalt der Völker auf dem Globus bekommt so eine klare Rangordnung in reiche und arme Nationen. Entwicklung ohne Vorherrschaft zu denken, macht deshalb genauso wenig Sinn, wie ein Wettrennen ohne Richtung abzuhalten.

Ferner kann man, zivilisationspolitisch betrachtet, die Entwicklung einer Nation an ihrem Grad der wirtschaftlichen Leistung ablesen, also am Bruttoinlandsprodukt. Gestützt auf eine ökonomische Weltsicht wie auf einen statistischen Werkzeugkasten definierten die Experten in den folgenden Jahrzehnten Entwicklung als Wachstum des Pro-Kopf-Einkommens. Gesell-

1 Wolfgang Sachs (Hg.), The Development Dictionary. A Guide to Knowledge as Power, London 1992. Der vorliegende Artikel ist das erweiterte Vorwort zu der Neuausgabe im Oktober 2019. Die deutsche Übersetzung damals war: Wie im Westen so auf Erden. Ein polemisches Handbuch zur Entwicklungspolitik, Hamburg 1993.

2 Einige andere Autoren zu dieser Zeit, die zu ähnlichen Thesen kamen, sind: Arturo Escobar, Encountering Development. The Making and Unmaking of the Third World, Princeton 1995, oder Gilbert Rist, The History of Development, London 1997. 
schaften, die soeben der Kolonisierung entronnen sind, sind gehalten, sich unter die Obhut der Ökonomie zu stellen.

Und schließlich, viertens, wenn man die Akteure betrachtet, sind die Impulsgeber der Entwicklung in erster Linie westliche Experten aus Regierungen, multinationalen Banken und Konzernen. Vormals, zu Zeiten von Marx oder Schumpeter, ist Entwicklung intransitiv gebraucht worden wie eine Blume, die einen Reifungszustand anstrebt und damit sich selbst entwickelt hat. Nun wurde der Begriff transitiv verstanden, als aktive Umgestaltung einer Gesellschaft, die innerhalb von Jahrzehnten, wenn nicht gar von Jahren zu bewerkstelligen war.

Was aber ist anschließend aus dieser Idee geworden? Sie hat einen Lauf genommen, wie er in der Ideengeschichte nicht ungewöhnlich ist: Was einmal eine historische Innovation war, wurde dann für lange Zeit zu einer Konvention, die schließlich in allgemeiner Frustration ihr Ende fand. Dennoch war es vor 30 Jahren ausgesprochen voreilig, das Ende des Entwicklungszeitalters zu behaupten. Denn die Entzauberung der Idee vollzog sich in Jahrzehnten - und sie ist heute noch nicht abgeschlossen.

\section{Vom Nationalstaat zur Globalisierung}

Seit dem Ende des Zweiten Weltkriegs war Entwicklung im Rahmen des Nationalstaats verstanden worden; gerade viele junge Nationen hatten ihre Staatsraison in der Entwicklung gesehen. Mit der Epoche der Globalisierung, ab der Zeitenwende von 1989/90, dem Ende der Bipolarität, war indessen der Nationalstaat porös geworden; der nationalstaatliche Behälter wurde nunmehr von globalen Kräften in Wirtschaft und Kultur durchlöchert. Güter, Geld, Informationen, Bilder, Menschen strömten über Grenzen hinweg und ließen einen transnationalen sozialen Raum entstehen, in dem Interaktion über große Distanzen erfolgt, manchmal sogar in Echtzeit. Der Nationalstaat fiel immer weiter zurück, andere Akteure spielten eine zunehmend wichtige Rolle. So überholten die privaten Auslandsinvestitionen die öffentliche Entwicklungshilfe, weltweit ausgestrahlte Fernsehbilder drängten nationale Erzählungen an den Rand und globaler Konsum löste das lokale Handwerk ab. Entwicklung, einstmals eine Staatsaufgabe, wurde somit deterritorialisiert und globalen Kräften unterworfen.

Außerdem traten transnationale Unternehmen auf den Plan, begleitet von einer sich globalisierenden Kulturindustrie. Allgemein gesprochen: Selbst in den entferntesten Winkeln der Erde ersetzte die kapitalistische Warenökonomie die zahllosen Subsistenzwirtschaften mit ihren traditionellen Märkten. Wobei sich auch der Kapitalismus verändert hatte, wie John Kenneth Galbraith schon am Ende der 50er Jahre analysiert hat: Von einer Wirtschaft, die der Bedarfsdeckung diente, zu einer Wirtschaft, die gerade aus der Bedarfsweckung ihren Profit erzeugte. Dabei geht es in einer solchen Wirtschaft zunehmend um die symbolische Macht der Güter und Dienstleistungen. Güter sind eher Kommunikationsmittel: Es zählt, was sie sagen, weniger 
was sie tun. Waren sind Rituale und Religion zugleich, und die Lebensstile gleichen sich an: SUVs lösen Rikschas ab, Smartphones treten an die Stelle von Dorfversammlungen, Air-Conditioning ersetzt die Siesta. Man kann die Globalisierung der Märkte als Entwicklung ohne Nationalstaaten verstehen.

Daraus hat die globale Ober- und Mittelklasse am meisten Nutzen gezogen, ob in Europa und Nordamerika oder, weniger zahlreich, in Lateinamerika und Afrika, aber ganz besonders in Asien. Gegenwärtig zählen um die drei Milliarden Menschen mit einem Einkommen von mehr als zehn Dollar zur Mittelklasse, vorwärtsgetrieben durch den steilen wirtschaftlichen Aufstieg in China, Indien und anderen asiatischen Ländern. Wobei die Beschleunigung besonders ins Auge fällt: 150 Jahre, beginnend mit der Industriellen Revolution, waren erforderlich, bis die erste Mittelklasse-Milliarde um das Jahr 1985 herangewachsen war; die zweite brauchte nur 21 Jahre und die dritte nur noch 9 Jahre; und wie Berechnungen ergeben, werden bis zum Jahr 2028 zwei weitere Milliarden hinzukommen. In der Summe sind das fünf Milliarden von neuen Verbrauchern, ${ }^{3}$ mit enormer Spreizung: Unten auf der Konsumleiter kann man sich (allenfalls) ein Moped oder eine Waschmaschine leisten, oben kann man in Fernreisen oder Immobilien investieren.

Hinter dem Verlangen nach Einkaufszentren und Autobahnen, Megawatts und Wachstumsraten steht indessen auch die Sehnsucht nach Anerkennung und Gerechtigkeit. Tief sitzen die Wunden, welche die jahrhundertelange koloniale Demütigung geschlagen hat. Der Erfolg der Mittelklasse besonders in den Schwellenländern ist eine Quelle von Stolz und Selbstachtung: Endlich ernst genommen zu werden unter den Mächtigen der Welt, ist eine Triebkraft der Entwicklung. Sie war sicher eine Erfindung des Westens, aber beileibe nicht nur ein Diktat an den Rest der Welt. Im Gegenteil, indem die Sehnsucht nach Anerkennung und Gerechtigkeit auf das Zivilisationsmodell des Nordens gerichtet war, stellten sich die Länder des Südens als überaus zähe Verfechter von Entwicklung heraus. All das ist fraglos ein grandioser Erfolg des Entwicklungsdenkens - doch im 21. Jahrhundert, das lehrt die aktuelle Kumulation der Krisen, können sich diesen Erfolg weder die Erde noch die auf und von ihr lebenden Menschen leisten.

\section{Der Niedergang der Entwicklungsidee}

Lange vorbei ist heute die Zeit, als Entwicklung noch Verheißung war. Damals war die Rede von jungen, aufstrebenden Nationen, die sich auf der Straße des Fortschritts befinden. Unbestritten erzeugte die Entwicklungsidee ein monumentales historisches Versprechen - das Versprechen, dass am Ende alle Gesellschaften die Kluft zu den reichen Nationen schließen könnten, um an den Früchten der industriellen Zivilisation teilzuhaben. Doch damit war spätestens vor fünf Jahren Schluss. 2015 ist ein Schlüsseljahr für das Entwicklungsdenken. Die Auflösung der Entwicklungsidee wurde greif-

3 Homi Kharas, The Unprecendeted Expansion of the Global Middle Class. An Update, Washington: Brookings Institution, Februar 2017, S. 1. 
bar in der im September 2015 verabschiedeten UN Agenda 2030, in der die Sustainable Development Goals (SDGs) festgelegt sind.

Überleben statt Fortschritt ist seither angesagt. Die SDGs sind darauf angelegt, das menschenrechtliche Minimum sowie das ökologisch Gebotene zu garantieren. Nicht mehr und auch nicht weniger, doch der himmelstürmende Fortschrittsglaube von einst hat damit dem schlichten Erfordernis der Überlebenssicherheit Platz gemacht. Die SDGs tragen zwar im Titel den Namen "development" - doch das ist eine semantische Täuschung. Die Sustainable Development Goals hätten in Wirklichkeit SSGs heißen müssen - Sustainable Survival Goals. Die im selben Jahr verabschiedete päpstliche Enzyklia "Laudato $\mathrm{si}^{\prime \prime}$ lässt denn auch den einstigen Schüsselbegriff Entwicklung gleich völlig außer Acht, während das am 12. Dezember 2015 vereinbarte Klimaabkommen von Paris davon zu retten sucht, was noch zu retten ist.

Dabei gibt es ja durchaus auch positive Tendenzen: Die Zahl der Armen ist global gesunken - doch diese Reduktion ist mit noch größerer Ungleichheit erkauft worden. Seit 1980 hat sich der Anteil der Einkommen der reichsten zehn Prozent massiv gesteigert, und zwar besonders in Nordamerika, China, Indien und Russland, weniger in Europa. ${ }^{4}$ Vor allen Dingen wurde auch die Nutzung der Erde drastisch überdehnt, nach den Berechnungen des Global Footprint Network verbraucht die Menschheit inzwischen jedes Jahr das 1,7fache der Biosphäre. Leergefischte Meere, durchlöcherte Tier- und Pflanzenwelt wie auch das Klimachaos legen davon Zeugnis ab.

Nicht zuletzt die Überhitzung der Erde und der Verschleiß der biologischen Vielfalt haben dem Glauben, dass die entwickelten Nationen die Spitze der sozialen Evolution sind, den Boden entzogen. Im Gegenteil, der Fortschritt hat sich in weiten Teilen als Rückschritt entpuppt, weil die Wirtschaftsweise des globalen Nordens gar nicht anders kann, als die Natur auszubeuten. Von den "Limits to Growth" von 1972 bis zu den "Planetary Boundaries" von 2009 sprechen die Analysen eine eindeutige Sprache: Entwicklung als Wachstum führt zur Unwirtlichkeit des Planeten Erde für den Menschen.

\section{Die geopolitische Implosion - das Ende von Nord und Süd}

Auch die der Entwicklung zugrunde liegende Geopolitik, ihre zweite Komponente, ist in den letzen 30 Jahren implodiert. Noch auf dem Millenniumsgipfel in New York im Jahr 2000 hatte man das Schema der vergangenen 50 Jahre reproduziert: die Welt, schön aufgeteilt nach Norden und Süden, wo sich die Geberländer zu den Empfängerländern beugten, um diese mit Kapital, Wachstum und Sozialpolitik für das globale Rennen wieder fit zu machen. In den Nachkriegsjahrzehnten war diese "nachholende Entwicklung“ in aller Munde gewesen. Was aber ist aus dem Imperativ des catching up geworden? Jenem Imperativ, der so fundamental ist für die Idee der Entwicklung? Dazu ist es wert, eine Passage des SDG-Dokuments zu zitieren. Dort heißt es:

4 World Inequality Report 2018, S. 12. 
„Diese Agenda ist von beispielloser Reichweite und Bedeutung. Sie wird von allen Ländern akzeptiert. [...] Ihre Ziele und Zielvorgaben sind universell und betreffen die ganze Welt, die entwickelten Länder wie die Entwicklungsländer." ${ }^{5}$ Die SDGs beanspruchen damit, global und universell, also für alle zu gelten. Deutlicher kann man den mentalen Bruch nicht formulieren: Die Geopolitik der Entwicklung, wonach die alt-industriellen Länder das Vorbild für die ärmeren Länder sein sollten, wurde feierlich entsorgt. Auch im Pariser Klimaabkommen von 2015 kommen „Entwicklungsländer" nur vor, wenn es um die Finanzierung der Anpassung an die Klimafolgen geht. Wie viel an Planung und Passion, wie viel an Ressourcen und Romantik hatte man doch aufgebracht, um den Traum des catching up zu verwirklichen! Nunmehr tempi passati: So wie 1989 die Ära des Kalten Krieges verwelkt war, so war 2015 der Mythos vom Aufholen dahin.

Selten wurde ein Mythos so formlos und noch dazu so geräuschlos beerdigt wie dieser. Und das durchaus zu Recht: Denn welchen Sinn macht Entwicklung noch, wenn es kein Land gibt, das man als nachhaltig entwickelt bezeichnen kann?

Auch die ökonomische Geographie der Welt hat sich fundamental verändert. Weltpolitisch zweifellos am spektakulärsten ist der rasante Aufstieg Chinas zur größten Wirtschaftsmacht der Erde. Doch dahinter kommt noch mehr zum Vorschein: Die sieben bedeutendsten Schwellenländer sind inzwischen wirtschaftlich stärker als die traditionellen Industriestaaten, die als die Gruppe der G 7 nur noch so tun, als hätten sie weiterhin die Hegemonie in der Weltwirtschaft. Es verringert sich also der Abstand zwischen den Nationen der Welt, wohingegen die Kluft innerhalb der Nationen zwischen Arm und Reich deutlich zugenommen hat. In anderen Worten, der Wettkampf der globalen Mittelklassen wird zumeist auf dem Rücken der mittel- und machtlosen Menschen betrieben. Seien es die Stauseen, Flurbereinigung oder Autobahnen: Überall wird oft die arme Mehrheit deklassiert. So hat die Globalisierung das hergebrachte Nord-Süd-Schema weitgehend aufgelöst.

\section{Entwicklung als statistisches Konstrukt}

Ferner war Entwicklung schon immer ein statistisches Konstrukt. Ohne die magische Zahl, das GDP, war man nicht imstande, eine Rangordnung unter den Nationen der Welt herzustellen. Einkommen zu vergleichen, das war die Pointe des Entwicklungsdenkens. Dabei erweist sich immer wieder, dass das Verhältnis von Nachhaltigkeit und wirtschaftlichem Wachstum ein dorniges, zutiefst widersprüchliches Feld ist. Die Agenda 2030 beklagt die gewaltige Ungleichheit auf der Welt, aber andererseits ruft sie nach Wirtschaftswachstum, für die ärmeren Staaten sogar nach einem von wenigstens 7 Prozent. Bewältigt werden soll der Widerspruch zwischen Nachhaltigkeit und Wachstum mit der Strategie des sogenannten inklusiven Wachstums.

5 Vereinte Nationen, Transformation unserer Welt: Agenda 2030 für eine nachhaltige Entwicklung. Beschluss der Vollversammlung, September 2015, Artikel 5. 
Dagegen pfeifen es die Spatzen von den Dächern, dass ein inklusives Wachstum, getrieben von den Finanzmärkten, ein Ding der Unmöglichkeit ist, weil es stets neue Ungleichheit produziert. Seit 1990 erlebten die Schwellenländer Russland, China, Indien, Südafrika einen steilen Anstieg der Ungleichheit. Die Schlussfolgerung liegt auf der Hand: Armutslinderung ohne Reichtumslinderung erweist sich als irrelevant.

Ähnliches gilt für die Losung vom grünen Wachstum. Dass die Schonung der terrestrischen wie marinen Ökosysteme mit der steten Wirtschaftsexpansion vereinbar sei, ist das bizarre Geheimnis der Agenda 2030. Es hat sich überdies schon rumgesprochen, dass das Wirtschaftswachstum, getrieben von fossilen Rohstoffen, angesichts der Grenzen der Erde mittelfristig ausgeschlossen ist. Deshalb fassten 2015 die Industriestaaten die Dekarbonisierung der Weltwirtschaft bis zum Jahrhundertende ins Auge. Europa nahm sich vor, bis 2050 „klimaneutral“ zu sein. Doch die Rezepte für grünes Wachstum setzen alle auf eine Entkopplung von Naturverbrauch und Wirtschaftswachstum, obwohl eine absolute Entkopplung, das heißt drastisch sinkender Ressourcenverbrauch auch bei steigendem BIP, in der neueren Geschichte noch nicht vorgekommen ist. Nicht viel spricht dafür, dass es in Zukunft anders sein wird. ${ }^{6}$ Doch davon, von der logischen Konsequenz, wagt die Agenda 2030 nicht zu sprechen: Wohlstand ohne Wachstum. Gewiss, wachsen sollen alle Sektoren, die der Nachhaltigkeit dienen - wie erneuerbare Energien, postfossile Mobilität, Biolandbau oder grüne Chemie. Aber schrumpfen müssen jene, die eine Gefahr für die Biosphäre darstellen - wie die fossil-energetischen, autoindustriellen und petrochemischen Komplexe. Es ist keineswegs sicher, dass die Summe der Wachstums- und Schrumpfungsprozesse zu einem dauerhaften aggregierten Wachstum führt. Den Wachstumszwang abzubauen, ganz besonders für die alten Industrieländer, das ist in der Agenda 2030 ein Tabu. Denn dafür hätte man die Effizienz der technischen Mittel klug mit der Suffizienz wirtschaftlicher Ziele kombinieren müssen. Papst Franziskus war damals jedenfalls schon weiter. In seiner Enzyklika „Laudato si“" empfiehlt er in Paragraph 193, an Degrowth für wohlhabende Zonen der Erde zu denken.

Schließlich haben sich die Akteure der Entwicklung anhaltend entzweit, ja häufig arbeiten sie gegeneinander. Schon seit den 1970er Jahren bildete sich eine Dichotomisierung des Entwicklungsdiskurses heraus, bei der die Idee der "Entwicklung als Wachstum" der Idee der "Entwicklung als Sozialpolitik" gegenübergestellt wurde. Institutionen wie die Weltbank, die Welthandelsorganisation oder die Asiatische Entwicklungsbank huldigten nach wie vor der Wachstumsidee, während sich das UNDP, UNEP und die meisten NGOs der Idee verschrieben, Entwicklung als Sozialpolitik zu denken. So aber wurde der Begriff Entwicklung zum Alleskleber, der die Anlage von Flughäfen ebenso umfasste wie das Bohren von Wasserlöchern.

Dazu kommt, dass nicht nur das Nord-Süd-Schema inzwischen hinfällig geworden ist, sondern auch das Ost-West-Schema. Seit 2010 haben die Inves-

6 Timothée Parrique u.a., Decoupling debunked: evidence and arguments against green growth as a sole strategy for sustainability, European Environmental Bureau, Brüssel, Juli 2019. 
titionen aus China im Süden der Welt jene aus den USA und Japan überholt. Ob Hafenanlagen in Kenia, Staudämme in Patagonien oder Gaspipelines in Turkmenistan: Überall errichtet heute China jene Infrastruktur, die früher der Westen gebaut hat. Und nicht nur ist der Einfluss des Westens kleiner geworden, sondern zugleich sind seine Selbstzweifel hinsichtlich seiner einstigen Mission des Westens größer, ja schier übermächtig geworden - bis hin zur offenen Leugnung. Der vorläufige Höhepunkt ist das Amerika des Donald Trump. Trump ist der erste Präsident - wie Ivan Krastev und Stephan Holmes $^{7}$ schreiben -, der Amerikas messianisches Selbstverständnis ablehnt, wonach die Vereinigten Staaten ein Leuchtfeuer der Freiheit und Gerechtigkeit für die gesamte Menschheit sind, ein Modell, nach dem alle Entwicklungsländer streben sollten.

\section{In der Zwickmühle: Entwicklung als Trugbild}

Wenn man über die Epoche nach der Entwicklung spricht, muss man immer wieder auf Mohandas (Mahatma) Gandhi zurückkommen, der Indien zur Unabhängigkeit geführt hat und dabei ein Vorläufer des Post-DevelopmentDenkens war. Er hinterließ der Nachwelt ein bekanntes Zitat, das sein Denken in Sachen Entwicklung lapidar zusammengefasst hat: "Die Welt hat genug für jedermanns Bedürfnisse, aber nicht für jedermanns Gier."

Schaut man das Zitat genauer an, so wird sein subversiver Charakterzug deutlich. Dann wird klar, warum Gandhi im gegenwärtigen Indien zwar als Säulenheiliger gilt, aber nur zu zeremoniellen Anlässen hervorgeholt wird.

Gandhi ist der Auffassung, dass die Ressourcen der Erde nicht knapp sind, im Gegensatz zum Lehrgebäude der Ökonomen, sondern vielmehr so reichlich, um den Bedarf der menschlichen Gesellschaft zu befriedigen. Dabei geht er davon aus, dass die Bedürfnisse kulturell geprägt, also durchaus endlich sind, ein weiterer Gegensatz zur ökonomischen Orthodoxie. Das erlaubt ihm, die Habsucht auf die Anklagebank zu setzen, denn die zum System gewordene Raffgier untergräbt die Bedürfnisse der Mehrheit der Menschen. Habsucht ist diejenige Variable, die darüber entscheidet, ob die Menschen genug zum Leben haben oder nicht.

Wenn die Autoren des Berichts der Brundtland-Kommission ihren Gandhi nur richtig gelesen hätten, als sie 1987 ihr Leitbild zur Nachhaltigen Entwicklung schufen! Dann hätte die klassische Definition der sustainable development nicht passieren dürfen: „als Entwicklung, die die Bedürfnisse der Gegenwart befriedigt, ohne zu riskieren, dass künftige Generationen ihre eigenen Bedürfnisse nicht befriedigen können“. Gandhi hätte insistiert, dass nicht alle Bedürfnisse gleichermaßen gültig sind; die oberen Zehntausend haben andersartige Bedürfnisse als die Mehrheit der armen Schlucker. So aber wurde in der Folgezeit die mangelnde Unterscheidung zwischen survival needs und luxury needs einer der Fallstricke dieser Definition. In

7 Ivan Krastev und Stephen Holmes, Das Licht, das erlosch, Berlin 2019. 
der Tat: Menschenrechte und Konsumrechte in einen Topf zu werfen, das ist das fatale Erbe des Entwicklungsbegriffs, der blind für Klassenverhältnisse ist. Wie kann man nur die sozialen Grundrechte auf Nahrung, Wohnen und Gesundheit auf demselben Niveau behandeln wie den Konsumanspruch auf SUVs, Immobilien und Aktien? Was haben die Mapuche in Südchile gemein mit den Wallstreet-Bankern oder die Baumwollarbeiter in Mali mit den StartUps in Shanghai? Nicht viel, außer dass sie womöglich im Trugbild der Entwicklung vereint sind. Doch da eröffnet sich ein Dilemma, das in diesem Trugbild stets verborgen geblieben ist. In relativ wohlhabenden Ländern, wo die physischen SDGs (Ernährung, Gesundheit, Energie) einigermaßen gesichert sind, also in Europa, Nordamerika, Japan, Argentinien, Chile oder Thailand, ist die Ökologie zerrüttet: Diese Länder überschreiten die planetarischen Grenzen und haben einen zu großen ökologischen Fußabdruck. ${ }^{8}$ Nimmt man die ideellen Indikatoren wie Gleichheit und Demokratie noch hinzu, sinkt bereits die Zahl der einschlägigen Länder, die jedoch in exzessiver Weise weit über die ökologischen Grenzen hinausgeschossen sind.

Umgekehrt gilt: Wo Länder in ihrem ökologischen Rahmen bleiben, sind die SDGs weitgehend unerfüllt. Es ist wie in einer Zwickmühle: Je höher der Lebensstandard eines Landes steigt, materiell, aber auch ideell, desto heftiger ist tendenziell der Verschleiß der Biosphäre. Und je weniger die sozialen Menschenrechte gesichert sind, desto kleiner ist der ökologische Fußabdruck - wenigstens was Emissionen und Materialien betrifft. Welch ein tragisches Resultat der Entwicklung!

Das außerdem noch schwerer wiegt, wenn das Wohlergehen der einen von der Armut der anderen abhängt. Beispiele dafür finden sich zuhauf: Lokale Fischer haben das Nachsehen, wenn große Fabrikschiffe die Weltmeere leerfischen; Kleinbauern werden vertrieben, wenn Agrarkonzerne massiv Land aufkaufen; Slumbewohner müssen weichen, wenn Stadtautobahnen gebaut werden; alteingesessene Bewohner werden ausquartiert, wenn die Gentrifizierung ihre Wohnviertel erreicht hat; Arbeiter werden Repressionen ausgesetzt, wenn sie ihre gewerkschaftlichen Rechte in Fabriken der globalen Wertschöpfungsketten geltend machen wollen. Mancherorts dringt die imperiale Lebensweise ${ }^{9}$ tief in die Lebensstile, Institutionen und Infrastruktur der Wohlhabenden ein. Unerkannt und doch wirksam durch eine Vielzahl von komplexen Wirtschaftsstrukturen und Ausbeutungsmechanismen, wird in der Summe ein Ergebnis erreicht: Die einen leben auf Kosten der anderen.

\section{Der Kampf der Narrative: Festungsdenken, Globalismus, Solidarität}

Angesichts dieser fatalen Bilanz werden die Problemlagen von Gesellschaften inzwischen nicht mehr primär als Probleme der Entwicklung aufgefasst,

8 Daniel W. O'Neill u.a., A good life for all within planetary boundaries, in: „Nature Sustainability", 1/2018, S. 88-95.

9 Ulrich Brand und Matthias Wissen, Imperiale Lebensweise. Zur Ausbeutung von Mensch und Natur im globalen Kapitalismus, München 2017. 
sondern die Debatten sind vielgestaltiger geworden und wandeln sich je nach den historischen Bedingungen. Wenn nicht alles täuscht, lassen sich gegenwärtig grob drei Erzählungen ausmachen: das Narrativ der Festung, das des Globalismus und das der Solidarität.

Das Festungsdenken lebt von einem Gemisch aus Nationalismus, Xenophobie sowie Autoritarismus und ist mit einer gehörigen Prise an Wohlstandschauvinismus gewürzt. Es bedient oft die populistische Sehnsucht nach einem "starken Mann", mit dem sich die sozial abgehängten Teile der Bevölkerung identifizieren können. Daneben begrüßen Nationalpopulisten vom Typus Trump oder Bolsonaro im Allgemeinen das Bohren nach Erdöl im Meer, das Fracking, den Kohlenabbau und die Abholzung der Wälder. Sie halten den Klimawandel für eine fein gesponnene Hinterlist der Feinde der nationalen Wirtschaft. Sie sind derart rückwärtsgewandt, dass sie die Ausplünderung der Natur regelrecht verherrlichen.

Dagegen dreht sich das Globalismus- oder Globalisierungsnarrativ um das Bild vom blauen Planeten als archetypisches Symbol. Anstelle des Merkantilismus des Festungsdenkens - America first - fordern die Globalisten eine deregulierte Freihandelswelt, die den Unternehmen sowie Konsumenten weltweit Profit und Wohlstand bescheren soll. Verglichen mit der Politik des Neoliberalismus geben sie öffentlichen Investitionen, auch in den Sozialstaat, mehr Raum und sehen allgemein eine größere Rolle für die Politik vor. International ist die multilaterale Governance die Devise. Allerdings halten die Globalisten das gegenwärtige Wirtschaftssystem nicht für nachhaltig. Geboten ist in ihren Augen ein Wirtschaftswachstum im Rahmen einer Grünen Ökonomie, worin besonders der Technologie die Aufgabe zukommt, mit den Umweltfolgen fertig zu werden. Die UN Agenda 2030 mit den Sustainable Development Goals fügt sich weitgehend in diesem Denkrahmen.

Davon radikal verschieden ist das Narrativ der Solidarität. Die öko-solidarische Ethik steht in Opposition sowohl zum Festungsdenken als auch zum Narrativ des Globalismus. Sie sieht eine postkapitalistische Ära voraus, gestützt auf einen Trend zur Öko-Solidarität, der auch die Regeln des Staates und der Wirtschaft umfasst. Die Monokultur der Ökonomie, die große Teile der Welt dominiert, könnte und müsste in dieser Weltsicht Platz machen für eine Vielzahl zivilisatorischer Alternativen, sei es die Weltsicht des Ubuntu oder des Buen vivir, sei es die Denkweise des europäische Humanismus oder der ahimsa in Jain-Tradition. ${ }^{10}$ Im Narrativ der Solidarität will man sowohl einen kulturellen als auch einen technischen Wandel betreiben, untermauert von kooperativen Wirtschaftsformen und gemeinwohlorientierter Politik. Darüber hinaus strebt man, wie es im Slogan "global denken, lokal handeln" zum Ausdruck kommt, einen kosmopolitischen Lokalismus an, wonach die lokale bzw. nationale Politik auch die Bedürfnisse der transnationalen Gemeinschaft berücksichtigen muss. Dies bedeutet, die imperiale Lebensweise des Nordens abzuwickeln und Land, Nahrung und Kapital in den Händen des globalen Südens zu belassen. Angesichts des Zerfalls der Ökosysteme

10 Ashish Kothari, u.a., Pluriverse. A Post-Development Dictionary, Neu-Delhi 2019. 
ist es besonders unerlässlich, die Ressourcenbasis des kapitalistischen Wirtschaftssystems radikal umzubauen: von fossilen zu solaren Ressourcen. Damit würde die Energie aus Wind, Sonne, Meer und Erdwärme gewonnen, während die regenerative Landwirtschaft Nahrung, Rohstoffe und Materialien bereitstellt. Statt weiter auf expansive Modernität für die globale Ober-/Mittelklasse zu setzen, ist es Zeit für eine reduktive Modernität (Harald Welzer): grüne Unternehmen, Zero-Emission-Häuser, viel weniger (nach den europäischen Standards) motorisierter Verkehr, viel weniger Fleischkonsum.

Entscheidend dafür ist es schließlich, neue Formen des frugalen Wohlstands herauszubilden: Zeitwohlstand statt Güterwohlstand, Sorgearbeit statt Lohnarbeit, Teilen statt Besitzen. Die entschleunigten Erfahrungen der Corona-Zeit könnten in dieser Hinsicht im besten Fall ein Vorschein auf einen anderen, nachhaltigeren Lebensstil sein.

\section{Entwicklung als Schlüsselwort nur mehr für Ideenhistoriker?}

Womöglich wird der Streit über diese drei Narrative über Jahre hinaus die Tagesordnung bestimmen. Dabei könnte Entwicklung, ähnlich wie Monarchie oder Feudalismus, immer weiter in den Dunst der Geschichte rücken, so weit, dass das einstige Schlüsselwort nur mehr für Ideenhistoriker von Interesse ist. Andererseits befinden wir uns derzeit, wenn nicht alles täuscht, in einer regressiven Epoche, in der der Begriff der Entwicklung wieder einmal fröhliche Urständ feiert. Die Trumps und Bolsanaros, die Erdoğans und Modis dieser Welt schwärmen für Entwicklung, sie glauben noch an Megaprojekte, Massenkonsum und freie Fahrt für Konzerne. Allerdings spielt bei den Nationalpopulisten die Idee der Entwicklung keine begeisternde Rolle mehr, wie zu den Zeiten der Dekolonisierung der jungen Nationen und auch noch zu den Zeiten der Deregulierung globaler Märkte. Ihr Bild von Entwicklung ist vielmehr stark autoritär geprägt und hat einen seltsam aggressiven Charakter. Die Nationalpopulisten sind ebenso glühende Anhänger der braunen Ökonomie wie eingeschworene Feinde der grünen Ökonomie. Ihre Entwicklungs- „Idee“ ist vor allem auf den Extraktivismus von Rohstoffen gebaut, sowohl von Kohle, Öl, Gas und Uran, als auch von Kaffee, Soja, Palmöl oder Tropenholz, so wie es in den Hochzeiten der Entwicklung 1960 bis 1990 üblich war. Indem sie das alte Industriesystem glorifizieren, stecken sie tief in der fossilistischen Vergangenheit fest und ahnen dabei nicht einmal, dass sie völlig zukunftsblind sind.

Freilich ist bei den Nationalpopulisten eine entscheidende Diskontinuität im Entwicklungsdenken zu verzeichnen: Sie erweisen sich als ethnozentriert und egoistisch. Während seit dem Zweiten Weltkrieg Entwicklung immer multilateral, im Guten wie im Bösen, gedacht worden war, hat sich spätestens mit dem Amtsantritt von Donald Trump der Wind radikal gedreht. "My country first" heißt bis auf weiteres der Kampfspruch des Unilateralismus als Entwicklung nur der eigenen Nation wie zur aggressiven Abwehr aller anderen. 